\title{
Surgical Treatment of Malignant Tumors of the Right Heart
}

\author{
Hiroshi Kubota, ${ }^{1} \mathrm{MD}$, Shinichi TAKAmoto, ${ }^{2} \mathrm{MD}$, Yutaka KotsuKa, ${ }^{2} \mathrm{MD}$, \\ Tsuyoshi MIYAIRI, ${ }^{1} \mathrm{MD}$, Tomohiro MURAKAWA, ${ }^{2} \mathrm{MD}$, Haruo MAKUUCHI, ${ }^{3} \mathrm{MD}$, \\ Motohiro KawAuchi, ${ }^{4} \mathrm{MD}$, Akira Furuse, ${ }^{4} \mathrm{MD}$, and Kenichi Sudo, ${ }^{1} \mathrm{MD}$
}

\section{SUMMARY}

Seven patients with malignant cardiac tumors were treated surgically in the Department of Cardiothoracic Surgery of the University of Tokyo between 1981 and 2000. Their treatments and outcomes are summarized and discussed. The ages of the patients ranged from 21 to 70 years old (mean: 49.5 \pm 15 ) and there were three males and four females. The histopathological diagnoses were hepatocellular carcinoma (HCC), spindle cell sarcoma, round cell sarcoma, osteosarcoma, renal cell carcinoma, and leiomyosarcoma. In four of the cases, the tumor extended or metastasized from other organs, while in the other three cases it originated in the heart. Before the cardiac operation, an above-knee amputation, left nephrectomy, transarterial embolization, or extended right hepatic lobectomy had been performed to treat the primary site of the tumor. Tumor resection using cardiopulmonary bypass was performed in every case. The NYHA classification of heart failure was significantly improved (preop: $3.3 \pm 0.8$, postop: $1.9 \pm 0.7[P<0.001]$ ). The mean survival period of the patients who died was $8.8 \pm 7.0$ months. A patient with renal cell carcinoma is still alive after 87 months of follow-up.

In summary, surgical treatment of malignant tumors of the right heart can improve the QOL in patients with cardiac failure. However, its effectiveness was temporary in all cases except one case of renal cell carcinoma. (Jpn Heart J 2002; 43: 263-271)

Key words: Cardiac tumor, Sarcoma, Round cell sarcoma, Spindle cell sarcoma, Leiomyosarcoma, Hepatocellular carcinoma, Renal cell carcinoma, Pulmonary embolism, Surgical treatment

TUMORS of the right heart have more diverse histological features and prognoses than tumors of the left heart. In this paper, we report on the clinical features and results of surgical treatment of patients with malignant tumors of the right heart encountered at the Department of Cardiothoracic Surgery of the University of Tokyo.

From ${ }^{1}$ Department of Cardiovascular Surgery, University of Kyorin. ${ }^{2}$ Department of Cardiothoracic Surgery, University of Tokyo, ${ }^{3}$ Toranomon General Hospital, ${ }^{4}$ JR Tokyo General Hospital, Tokyo, Japan.

Address for correspondence: Hiroshi Kubota, MD, Department of Cardiovascular Surgery, University of Kyorin. 6-20-2, Shinkawa, Mitaka-shi, Tokyo, 181-8611, Japan.

Received for publication March 15, 2001.

Revised and accepted February 22, 2002. 


\section{Patients}

The subjects were 7 patients with malignant tumors of the right heart seen between 1981 and 2000. They consisted of 3 males and 4 females, ranging in age from 21 to 70 years (mean: $49.5 \pm 15$ years) (Table I). The tumor was histologically diagnosed as hepatocellular carcinoma (HCC) in 2 cases, and spindle cell sarcoma, leiomyosarcoma, round cell sarcoma, osteosarcoma, and renal cell carcinoma in one case each.

In 2 cases (case 1: spindle cell sarcoma and case 3: round cell sarcoma), the primary site of the tumor was the right atrium. In case 2: leiomyosarcoma, the tumor was confined to the pulmonary artery. In 4 cases (case 4-7), the tumor had extended or metastasized from other organs (Figure. 1).

There was no significant difference between the mean age of the patients with a primary cardiac tumor and those with a metastatic cardiac tumor $(57 \pm 11$ years vs. $44 \pm 27$ years, $P=0.30$ ). No symptoms specific to histological diagnosis were observed before the surgery. Symptoms and signs of right heart failure were the most frequent presenting features. All of the patients had dyspnea. Other symptoms included exertional palpitations, tachycardia, edema of the face and leg, pleural effusion, ascites, cough, and shock. A patient with spindle cell sarcoma went into shock during transportation from another hospital. A patient with leiomyosarcoma of pulmonary artery origin suffered from recurrent episodes of syncope which might have been caused by hypoxia. In the patient with renal cell carcinoma, massive pulmonary emboli in the bilateral pulmonary artery, which was separate from the local extension to the inferior vena cava, caused severe shortness of breath and shock.

The metastatic routes to the right heart of tumors originating from other organs were as follows. In case 5, the HCC had metastasized without continuity to the right ventricular outflow tract and the pulmonary artery, while in case 4 , the

Table I. Clinical Characteristics of the Patients

\begin{tabular}{cclll}
\hline Case No. & Age and Sex & \multicolumn{1}{c}{ Pathological Dx. } & Primary site & \multicolumn{1}{c}{ Preoperative symptoms } \\
\hline 1 & $51 \mathrm{~F}$ & Spindle cell sarcoma & RA & Dyspnea, edema, ascites, shock \\
2 & $50 \mathrm{~F}$ & Leiomyosarcoma & PA & Dyspnea, syncope \\
3 & $70 \mathrm{~F}$ & Round cell sarcoma & RA & Dyspnea, edema, pleural and pericardial effusion \\
4 & $47 \mathrm{~F}$ & Hepatocellular carcinoma & Liver & Dyspnea \\
5 & $48 \mathrm{~F}$ & Hepatocellular carcinoma & Liver & Dyspnea, dizziness \\
6 & $21 \mathrm{M}$ & Osteosarcoma & Fibula & Dyspnea, tachycardia \\
7 & $60 \mathrm{M}$ & Renal cell carcinoma & Lt.Kidney & Dyspnea, shock \\
\hline
\end{tabular}




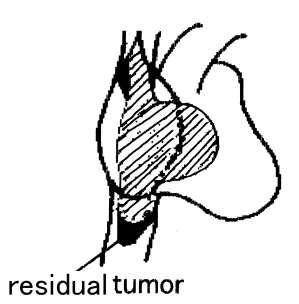

1 Spindle cell sa.

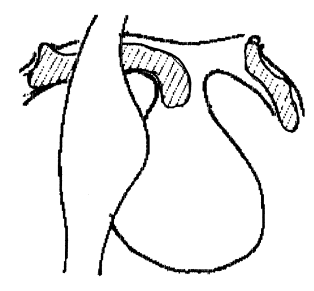

2 Leiomyosarcoma

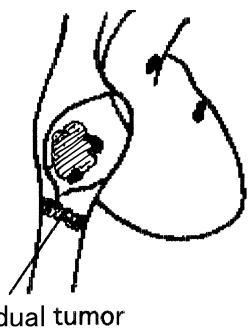

3 Round cell sa.

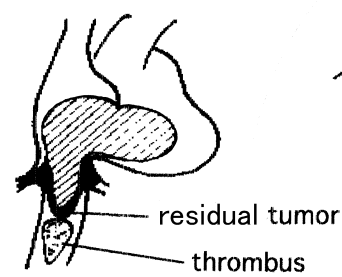

$4 \mathrm{HCC}$

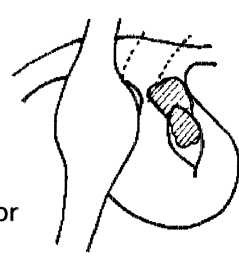

$5 \mathrm{HCC}$

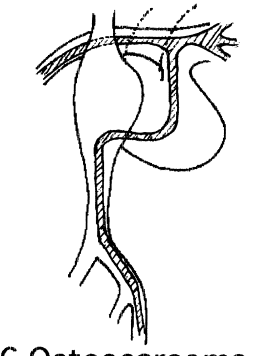

6 Osteosarcoma

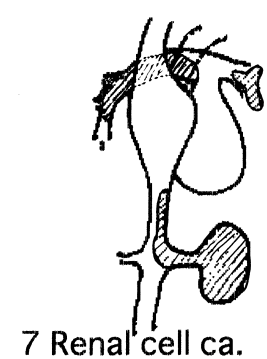

7 Renal cell ca.

Figure 1. Location of the tumor. sa.=sarcoma; ca.=carcinoma; $\mathrm{HCC}=$ hepatocellular carcinoma.

HCC had extended to the inferior vena cava, right atrium, and then to the right ventricle. In case 6: osteosarcoma, the tumor extended via the veins draining the fibula to the inferior vena cava, right atrium, right ventricle, and then the pulmonary artery. In case 7, the renal cell carcinoma extended to the inferior vena cava. A part of the extended tumor separated and went into the pulmonary artery (Table II, Figure. 1).

The primary tumors other than those of the heart were treated as follows: In case 4, extended right lobectomy of the liver was performed. In case 5, transarterial embolization with MMC and Lipiodol was applied. In case 6, an above-knee leg amputation was performed. In case 7 , left nephrectomy+resection of a tumor in the inferior vena cava+regional lymph node excision were performed (Table II).

The preoperative NYHA grade was IV in 3 cases, III in 3 cases, and II in 1 case (mean: $3.3 \pm 0.8$ ). 
Table II. Cardiac Malignant Tumor Extended or Metastasized from Other Organs

\begin{tabular}{cllll}
\hline Case & $\begin{array}{c}\text { Pathological } \\
\text { diagnosis }\end{array}$ & Origin & \multicolumn{1}{c}{ Extension } & \multicolumn{1}{c}{ Treatment } \\
\hline 4 & HCC & Liver & ...RV-PA & Extended Rt. Lobectomy \\
5 & HCC & Liver & -IVC-RA-RV & TAE (MMC+Lipiodol) \\
6 & Osteosarcoma & Fibula & -IVC-RA-RV- & Above-knee amputation \\
7 & Renal cell ca. & Lt. Kidney & PA & Lt. Nephrectomy+IVC Tumorectomy \\
& & & -IVC...RA & \\
\end{tabular}

$\mathrm{HCC}=$ hepatocellular carcinoma; $\mathrm{IVC}=$ lnferior vena cava; $\mathrm{RA}=$ right atrium; $\mathrm{RV}=$ right ventricle; TAE $=$ Trans arterial embolization.

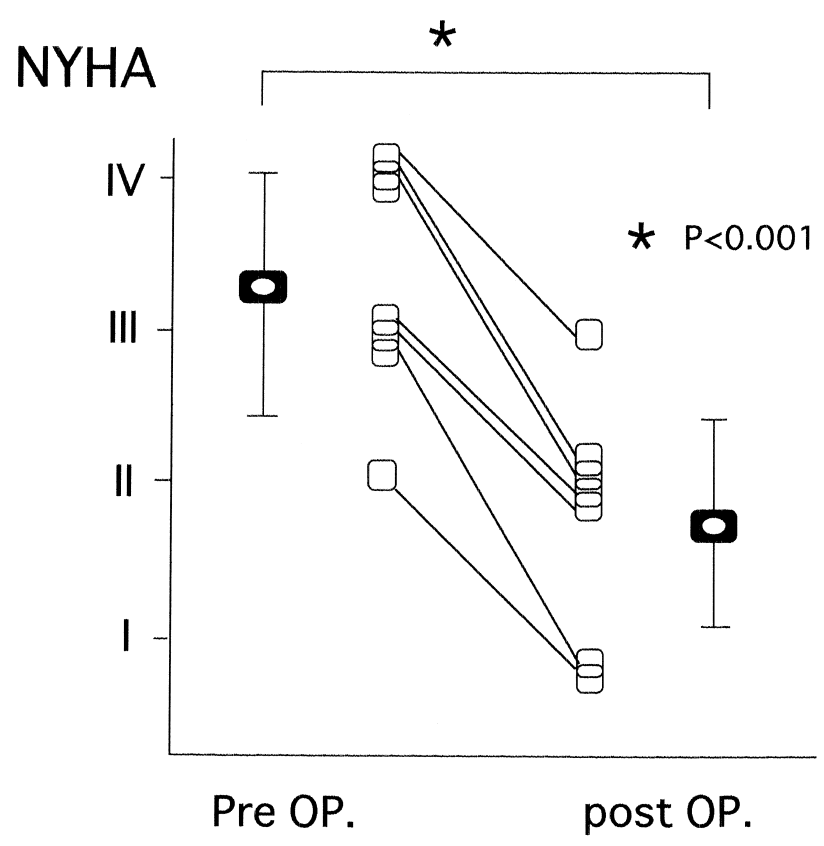

Figure 2. New York Heart Association functional classification before and after surgery.

\section{RESULTS}

In all cases, a cardiopulmonary bypass was employed to resect the tumor. The patient with spindle cell sarcoma underwent emergency surgery. The other patients underwent elective surgery. The cannulations of the cardiopulmonary bypass were done via the ascending aorta and the superior and inferior vena cava (via the femoral vein in cases where the tumor filled the right atrium or extended 
into the inferior vena cava). We attempted to remove the tumor as completely as possible, however, complete tumor resection was not possible in the cases with round cell sarcoma, spindle cell sarcoma, and HCC (Figure 1). After the surgery, the symptom improved in all case, and the NYHA grade also improved significantly (mean: $3.3 \pm 0.8$ to $1.9 \pm 0.7, P<0.001$ ) (Figure 2). However, in 6 cases, the effectiveness of the surgery was not maintained for very long. Despite treatment in the case with spindle cell sarcoma (case 1), in whom the tumor had filled the right atrium and invaded the inferior vena cava, it was impossible to resect the tumor completely. Although the patient's symptoms resolved partially for awhile after the surgery, she died of heart failure 3 months later, and was judged as having improved little as a result of surgery. The patient with leiomyosarcoma (case 2 ) died of local recurrence 3 months after surgery. The patient with round cell sarcoma (case 3 ) also showed a temporary resolution of symptoms, but her respiratory function became compromised suddenly 2 weeks after the surgery, necessitating re-intubation. This patient died of DIC 7 months after the surgery and was judged as having improved little as a result of the surgery. In the HCC case in which the tumor had directly invaded the heart (case 4), severe dyspnea was noted before the surgery, but the symptom resolved after the surgery and the patient was able to return to work for awhile. In case 5, the right ventricular metastasis of the HCC showed local recurrence. She underwent reoperation 5 months after the initial operation. However, complete resection of the recurrent tumor was impossible. Her symptoms resolved and her QOL improved after each operation, but she died of brain and lung metastases 10 months after surgery. In case 6 , three additional operations were conducted to treat residual or pulmonary metastatic tumors, however, the patient died of lung metastasis 1 year and 10 months after the cardiac operation (Figure 3). The mean postoperative survival

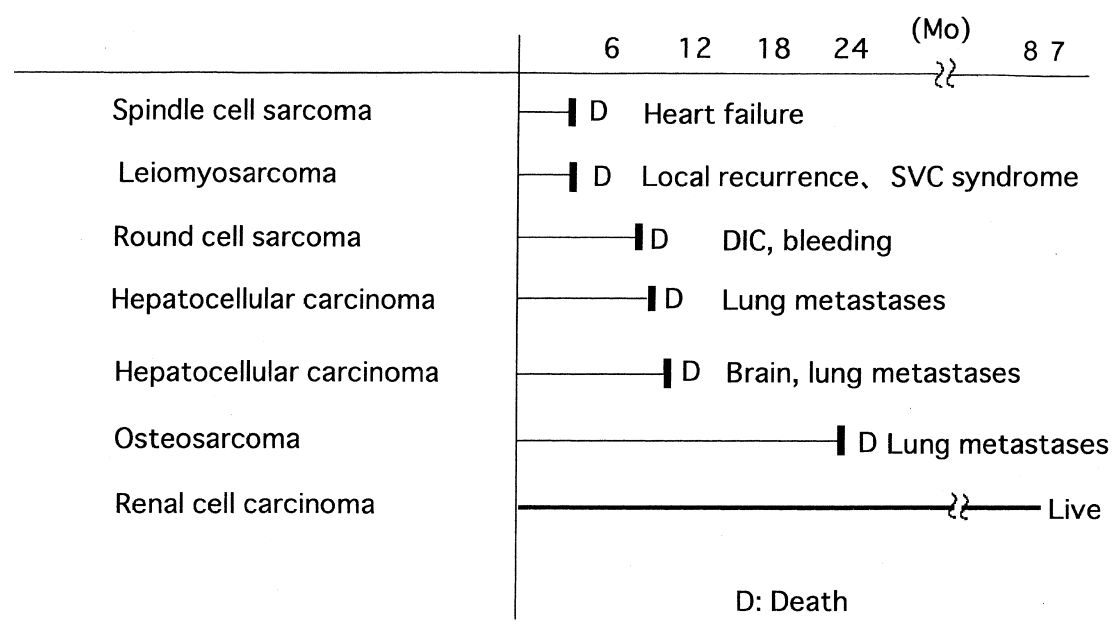

Figure 3. Postoperative clinical courses of the patients. 
period of the patients who died was $8.8 \pm 7.0$ months. Only the patient with renal cell carcinoma with massive pulmonary embolism caused by a tumor embolus is alive at the present time and free of local recurrence and lung metastasis seven years and three month after the operation. Although there was no significant difference, the postoperative survival period in the patients with a primary cardiac tumor tended to be shorter than that of those with a metastatic tumor $(4.3 \pm 2.3$ months vs $31.8 \pm 18.7$ months, $P=0.27$ ).

\section{DISCUSSION}

Primary cardiac tumors are infrequent. The majority of the tumors are benign. Perchnsky and colleagues reported a retrospective review of all patients with primary cardiac tumors managed by the University of British Columbia over a period of 40 years. ${ }^{1)}$ According to the report, 71 patients (25 males and 46 females) with primary cardiac tumors were identified. Fifty-seven $(80 \%)$ of the tumors were benign masses and $14(20 \%)$ were malignant masses. Twenty-eight percent of the tumors in men were malignant ( 7 of 25 ) compared with only $15 \%$ in women (7 of 46). Seventy-two percent of the masses were located in the left atrium. Forty-three percent of the tumors in the right atrium were malignant (6 of 14) compared with only $14 \%$ in the left atrium ( 7 of 51). Complete resection was possible for 50 of 52 benign atrial tumors (96\%) but for only 5 of 14 malignant tumors $(36 \%)$. Twelve patients died of their disease. They also mentioned that malignant cardiac tumors tend to be diagnosed after they have become extensive and the patient already has a very grave prognosis. Our three cases of primary cardiac tumor were also diagnosed late, showed a very poor prognosis, and the effect of surgical resection was only temporary.

The incidence of metastatic malignant tumors of the heart is estimated to be between $10.0 \%$ and $15.4 \%$, based on an analysis of autopsy findings in which a malignant neoplasm was diagnosed; the most frequent site of the primary tumor is the lung. The gastrointestinal system, genitourinary system, and skin (in particular, primary malignant melanoma) also constitute frequent sites of primary tumors that metastasize to the heart. ${ }^{2)}$ Metastasis of HCC to the heart has been reported in autopsied cadavers, but it is very rare. ${ }^{3)}$ Our cases of HCC died of lung metastasis or brain metastasis. We believe that their prognosis improved as a result of our surgical intervention, considering that death from heart failure was prevented. ${ }^{4)}$

To date, about 120 cases of sarcoma of the pulmonary artery have been reported. Leiomyosarcoma accounted for only 22 of these cases. Leiomyosarcoma affects women more frequently than men, and in more than $90 \%$ of the cases, this sarcoma develops in the main trunk of the pulmonary artery. ${ }^{5)}$ The 
prognosis is very poor. The survival period after diagnosis has been reported to be 1.5 months on average. The postoperative survival period of patients with this sarcoma has been reported to be 10 months. ${ }^{6}$ Because tumors within the pulmonary artery and tumor emboli cause symptoms such as progressive dyspnea and hypoxemia, which resemble the symptoms of chronic thromboembolism of the pulmonary artery, it is often difficult to make a definitive diagnosis based on the clinical findings alone. It has been reported that preoperative diagnosis of these tumors may be possible by histological examination of intravenous aspiration biopsy specimens, ${ }^{7)}$ and by checking for a homogeneous intermediate signal intensity on T1-weighted magnetic resonance images. ${ }^{8)}$ If detected in the very early stages, treatment of these tumors with vascular prostheses or homografts may be somewhat successful, but no long-term survivors have been reported. Since the effectiveness of chemotherapy or radiotherapy for these tumors has not been established, it is unknown to what extent surgical treatment contributed to prolonging the survival in our patients. ${ }^{9)}$

Our patient with renal cancer presented with clinical features different from those of the other patients with malignant tumors. Marshall, et al reported that among patients with renal cancer, 33\% show tumor spread to the renal vein and $6 \%$ exhibit tumor invasion of the inferior vena cava. ${ }^{10)}$ According to Javidan, et al the survival rate of patients with renal cancer is $95 \%$ for cases with stage I cancer, $88 \%$ for those with stage II, $59 \%$ for those with stage III, and $20 \%$ for those with stage IV cancer. ${ }^{11,12)}$ In our cases, preoperative pulmonary blood flow scintigraphy revealed multiple defects in both lung fields, and CT revealed multiple infarctions in the lungs; no signs of lung metastasis were observed. In cases with renal cancer, caution must be used to avoid misdiagnosing lung infarction as lung metastasis. The tumor in the present case was postoperatively rated as T3b, N0, M0, and Stage III. If lung metastasis was present, the tumor would have been classifed into stage IV, and the prognosis and treatment methods would have been different. Wagner, et al report that pulmonary infarcts can "mimic" pulmonary metastases originating from renal cancer. Masumori, et al reported two cases of renal cancer in which defects revealed by pulmonary blood flow scintigraphy later disappeared, thereby allowing a diagnosis of lung metastasis to be excluded. As is evident from these reports, pulmonary infarction detected in cases of renal cancer can be deemed as representing lung metastasis only if certain conditions are met, and this diagnostic confusion may be the reason why the reported clinical features of renal cancer differ from those of other malignant tumors. ${ }^{13-16)}$

Needless to say, the pathophysiological features and prognosis of malignant tumors can vary depending on their histological type. In cases of cancer in the right heart, signs of right heart failure are commonly seen. The treatment strategy in all these cases is the same in that attempts are made to resect the tumor as com- 
pletely as possible using a cardiopulmonary bypass.

The results of treatment of malignant tumors of the right heart system are unsatisfactory, unlike those of benign tumors in the same region. ${ }^{17)}$ The postoperative survival period of the patients who died was only $8.8 \pm 7.0$ months. In addition to control of the primary site of the tumor, advanced procedures such as heart transplantation, heart-lung transplantation, replacement using a vascular homograft, implantation of autologous cultured myocardium, or cardiomyoplasty might improve the prognosis of a malignant cardiac tumor.

\section{CONCLUSION}

Surgical treatment of malignant tumors of the right heart can improve the QOL in patients with cardiac failure, however, its effectiveness was temporary in all cases except one case of renal cell carcinoma. An early diagnosis and advanced treatment may improve the prognosis.

\section{REFERENCES}

1. Perchinsky MJ, Lichtenstein SV, Tyers GFO. Primary cardiac tumors - Forty years' experience with 71 patients. Cancer 1997; 79: 1809-15.

2. Klatt EC, Heitz D. Cardiac metastasis. Cancer 1990; 65: 1456-9.

3. Masaki N, Hayashi S, Maruyama T, et al. Marked clinical improvement in patients with hepatocellular carcinoma by surgical removal of extended tumor mass in right atrium and pulmonary arteries. Cancer Chemother Pharmacol 1994; 33 (Suppl): S7-S11.

4. Murakawa M, Takamoto S, Ezure M, Ono M, Kawauchi M, Tanaka O. Metastatic hepatocellular carcinoma obstructing the right ventricular outflow tract. Jpn J Thorac Cardiovasc Surg 2000; 48; 516-9.

5. Dumont P, Diot P, Aupart MR, Toumieux B. Leiomyosarcoma of the pulmonary artery. Ann Thorac Surg 1998; 66: 2089-91.

6. Kruger I, Borowski A, Horst M, deVivie ER, Theissen P, Gross-Fengels W. Symptoms, diagnosis, and therapy of primary sarcomas of the pulmonary artery. Thorac Cardiovasc Surg 1990; 38: 91-5.

7. Yamada N, Kamei S, Yasuda F, Isaka N, Yada I, Nakano T. Primary leiomyosarcoma of the pulmonary artery confirmed by catheter suction biopsy. Chest 1998; 113:555-6.

8. Blum U, Wildanger G, Windfuhr M, Laubenberger J, Freudenberg N, Munzar T. Preoperative CT and MR imaging of inferior vena cava leiomyosarcoma. Eur J Radiol 1995; 20: 23-7.

9. Kubota H, Takamoto S. A 50-year-old woman complaining of severe dyspnea. J Cardiol 1999; 34: 93-5.

10. Marshall VF, Middleton RG, Holswade GR, Goldsmith EI. Surgery for renal cell carcinoma in the vena cava. J Urol 1970; 103: 414-20.

11. Javidan J, Stricker HJ, Tamboli P, et al. Prognostic significance of the 1997 TNM classification of renal cell carcinoma. J Urol 1999; 162: 1277-81.

12. Sobin LH, Wittekind Ch. International Union Against Cancer (UICC): TNM classification of malignant tumors, 5th ed. New York: Wiley-Liss Publications, 1997; 180-2.

13. Masumori N, Iwabe H, Kumamoto E, et al. Studies on pulmonary metastasis of renal cell carcinoma-pulmonary embolism revealed by lung perfusion imaging and the metastasis [in Japanese]. Nippon Hinyokika Gakkai Zasshi 1991; 82: 769-75.

14. Wagner JR, Merino MJ, Pass HI, Linehan WM, Walther MM. Pulmonary infarcts can mimic pulmonary metastases from renal cancer. J Urol 1997; 158: 1688-90. 
15. Kubota H, Furuse A, Kotsuka Y, Yagyu K, Kawauchi M, Saito H. Successful management of massive pulmonary tumor embolism from renal cell carcinoma. Ann Thorac Surg 1996; 61: 708-10.

16. Kubota H, Takamoto S, Kotsuka Y, Furuse A. Successful treatment of massive pulmonary tumor embolism from renal cell carcinoma. Ann Thorac Surg 2000; 69: 972-3.

17. Kotsuka Y, Furuse A, Yagyu K. et al. Long-term results of surgical treatment of intracardiac tumors - Effectiveness and limitation of surgical treatment. Jpn Heart J 1995; 36: 213-23. 